\title{
The pulmonary embolism after switching from letrozole to tamoxifen in a patient with breast cancer. A case report and literature review
}

Agata Tomaszewska, Małgorzata Żach-Muzolf, Radosław Grabysa

Department of Internal Medicine, Warmian-Masurian Oncology Centre, Olsztyn, Poland

Correspondence:

Agata Tomaszewska Department of Internal Medicine, Warmian-Masurian Oncology Centre, Olsztyn, Poland 10-228 Olsztyn, al. Wojska Polskiego 37 e-mail: tomaszewskaagata1@gmail.com tel.: +48 794190135

Received:

21.03.2020

Accepted:

31.03.2020

DOI: 10.24292/01.OR.120311320

Copyright $\odot$ Medical Education. All rights reserved.

\section{ABSTRACT}

Women with breast cancer are affected by deep venous thrombosis and/or pulmonary embolism 3 times more often than the group of healthy women in the same age. Thrombosis can be the first manifestation of a malignant disease, but it can also result from hormonal therapy with aromatase inhibitors or tamoxifen which is used in cancer treatment. The necessary clinical problem to solve in this population is the issue of prophylactic antithrombotic treatment. In this paper we present a case of pulmonary embolism in the course of the breast cancer, which was associated with tamoxifen hormone therapy and inadequate thromboprophylaxis.

Key words: breast cancer, tamoxifen, pulmonary embolism, thromboprophylaxis 


\section{INTRODUCTION}

Venous thromboembolism (VTE) is a leading, but potentially preventable cause of death worldwide [1]. The risk of developing deep venous thrombosis (DVT) and/or pulmonary embolism (PE) is 4-13 fold more frequent in patients with advanced cancer compared to the patients with early stages of the disease [2]. Of note, women with breast cancer are affected by these potentially fatal complications 3 times more often than the group of healthy women in the same age [3]. Nevertheless, despite the low relative risk, since breast cancer is one of the most prevalent cancers worldwide, VTE is commonly seen in this population. In that case, thrombosis can be the first manifestation of a malignant disease, but it can also result from endocrine therapy used in cancer treatment [4]. Hormonal therapy with tamoxifen or aromatase inhibitors (Al) is the cornerstone of adjuvant therapy for women with estrogen positive breast cancer. Antiestrogenic activity of these substances is responsible for VTE risk, primarily during treatment with tamoxifen [5].

\section{CASE PRESENTATION}

A 71-year-old woman with hypertension, hypothyroidism and history of non-resectable right breast cancer in the tail of Spence with the metastasis to bones and lungs was referred to the Internal Medicine Department at the Oncology Centre due to progressive weakness, shortness of breath and bone pain. The histopathology of right breast biopsy showed the invasive ductal carcinoma (grade II) with the immunochemistry positive profile for estrogen receptor (ER), progesterone receptor (PgR) and androgen receptor (AR). Due to the progression of the disease, she was disqualified from the mastectomy. Letrozole therapy has been initiated and continued for the next year. At the same time the patient presented symptoms such as severe bone pain, therefore she underwent a cycle of palliative radiotherapy after radiotherapy consultation. After about 11 months of surveillance osteoporotic fracture of the right humerus was diagnosed and treated with surgery. Due to severe osteoporosis and disease progression letrozole was switched to tamoxifen in the dosage of $20 \mathrm{mg}$ per day as a second-line treatment. After discharge no antithrombotic prevention was included in postoperative treatment.

During a standard follow-up conducted after 5 months of tamoxifen therapy a computed tomography (CT) of thorax was performed in order to identify any cancer progression. The angio-CT of pulmonary arteries revealed a massive thrombi located in the right pulmonary artery and partially blocked left pulmonary artery in its distal part (fig. 1).
FIGURE 1.

Computed tomography with contrast showing pulmonary emoblism at the time of diagnosis.

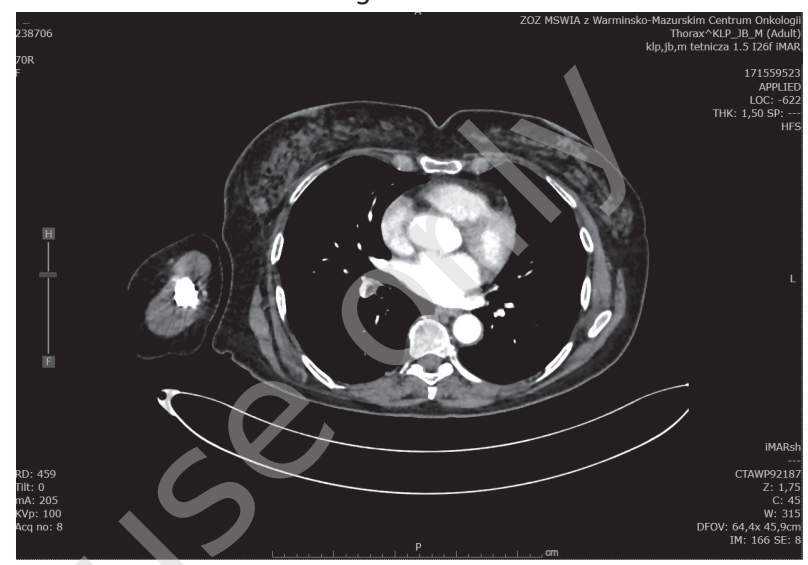

On the examination at admission to the Internal Medicine Department, the patient was in fair general condition, hemodynamically stable, afebrile, with blood pressure of $130 / 75 \mathrm{mmHg}$ and a regular, strong symmetrical pulse of $80 \mathrm{bpm}$. Cardiac and pulmonary auscultation revealed regular heart rhythm, normal heart and breath sounds. There were no hepatomegalia and lower limb edema.

Electrocardiogram demonstrated sinus rhythm with nonspecific ST-segment changes. Laboratory test results are shown in the table (tab. 1).

TABLE 1.

Patient's laboratory tests during initial hospitalization in the Internal Medicine Department.

\begin{tabular}{|c|c|c|}
\hline Laboratory test & Patient's result & Laboratory references range \\
\hline D-dimer & $4087,0 \mathrm{ng} / \mathrm{mL}$ & $500 \mathrm{ng} / \mathrm{mL}$ \\
\hline NT-proBNP & $153 \mathrm{pg} / \mathrm{mL}$ & $<125 \mathrm{pg} / \mathrm{mL}$ \\
\hline CRP & $7,84 \mathrm{mg} / \mathrm{L}$ & $<5 \mathrm{mg} / \mathrm{mL}$ \\
\hline $\begin{array}{c}\text { Capillary blood } \\
\text { gases }\end{array}$ & \multicolumn{2}{|l|}{} \\
\hline $\mathrm{pH}$ & 7,436 & $7,35-7,45$ \\
\hline $\mathrm{pCO}_{2}$ & $34 \mathrm{mmHg}$ & $35-45 \mathrm{mmHg}$ \\
\hline $\mathrm{pO}_{2}$ & $81,4 \mathrm{mmHg}$ & $65,0-95,0$ \\
\hline $\mathrm{HCO}_{3}$ act & $22,1 \mathrm{mmol} / \mathrm{L}$ & $21,0-27,0$ \\
\hline $\mathrm{BE}(\mathrm{B})$ & $-1,4 \mathrm{mmol} / \mathrm{L}$ & $-2,5-+2,5$ \\
\hline $\mathrm{O}_{2} \mathrm{SAT}$ & $96,6 \%$ & $70,0-95,0$ \\
\hline $\mathrm{CA}$ ionised & $1,313 \mathrm{mmol} / \mathrm{L}$ & $1,12-1,32 \mathrm{mmol} / \mathrm{L}$ \\
\hline Creatinine & $0,63 \mathrm{mg} \%$ & $0,50-0,90 \mathrm{mg} \%$ \\
\hline Protein C & $102 \%$ & $70-140 \%$ \\
\hline ACA & negative & negative \\
\hline ANA & negative & negative \\
\hline Hgb & $12,7 \mathrm{~g} / \mathrm{dL}$ & $11,5-15,7 \mathrm{~g} / \mathrm{dL}$ \\
\hline
\end{tabular}




\begin{tabular}{|c|c|c|}
\hline Laboratory test & Patient's result & Laboratory references range \\
\hline $\begin{array}{c}\text { ANTIi- } \beta_{2} \\
\text { GLYCOPROTEIN } \\
\text { I ANTIBODIES IgG/ } \\
\text { IGM }\end{array}$ & $\begin{array}{c}\text { negative/ } \\
\text { negative }\end{array}$ & negative/negative \\
\hline PLT & $199 \times 10^{3}$ & $150-450 \times 10^{3}$ \\
\hline TSH & $5,240 \mathrm{ulU} / \mathrm{mL}$ & $0,270-4,20 \mathrm{ulU} / \mathrm{mL}$ \\
\hline
\end{tabular}

NT-proBNP - N-terminal prohormone of brain natriuretic peptide, CRP - C-reactive protein, $\mathrm{pCO}_{2}$ - partial pressure of carbon dioxide, $\mathrm{pO}_{2}$ - partial pressure of oxygen, $\mathrm{BE}$ - base excess, ACA anti-centromere antibodies, ANA - anti-nuclear antibodies, $\mathrm{Hb}$ - hemoglobin, PLT - platelet count, TSH - thyroid-stimulating hormone.

A transthoracic echocardiography revealed: no signs of right heart pressure overload with the tricuspid pressure gradient (TVPG) reaching $25 \mathrm{mmHg}$ and pulmonary acceleration time (AcT) of $100 \mathrm{~ms}$ and normal, no cardiac chambers dilation and normal left ventricular systolic/diastolic function (left ventricular ejection fraction, LVEF 63\%).

Compression ultrasonography (CUS) of lower limb veins revealed deep vein thrombosis of the right lower limb veins and partial blockage in the distal fragment of the vessel of tight of the left lower limb.

Considering the overall clinical picture and the results of additional diagnostic tests (especially angio-CT of pulmonary arteries) initial diagnosis of pulmonary embolism due to deep vein thrombosis of the lower limbs during tamoxifen therapy was posed.

In this situation tamoxifen was withdrawn and treatment with low molecular weight heparin (enoxaparin) at a dose of $1 \mathrm{mg} / \mathrm{kg}$ twice a day was included.

During further hospitalization the patient was in good, stable condition, with no signs of hypotension or heart/respiratory failure. At the same time we observed gradual reduction of breathlessness with improving physical performance. After 7 days in good general condition, the patient was discharged with a recommendation to continue treatment with enoxaparin at a pre-determined dose for the next 4 weeks.

After this time she was presented to our institution for clinical reevaluation and determination of further antithrombotic and oncological treatment strategy. Control angio-CT of pulmonary arteries showed regression of thromboembolic lesions (fig. 2) observed previously. Finally, the patient was qualified for further, at least six-month treatment with enoxaparin and palliative radiotherapy due to bone pain.
FIGURE 2.

Computed tomography with contrast after 4 weeks of treatment with enoxaparin showing regression of thromboembolic lesions in pulmonary arteries.

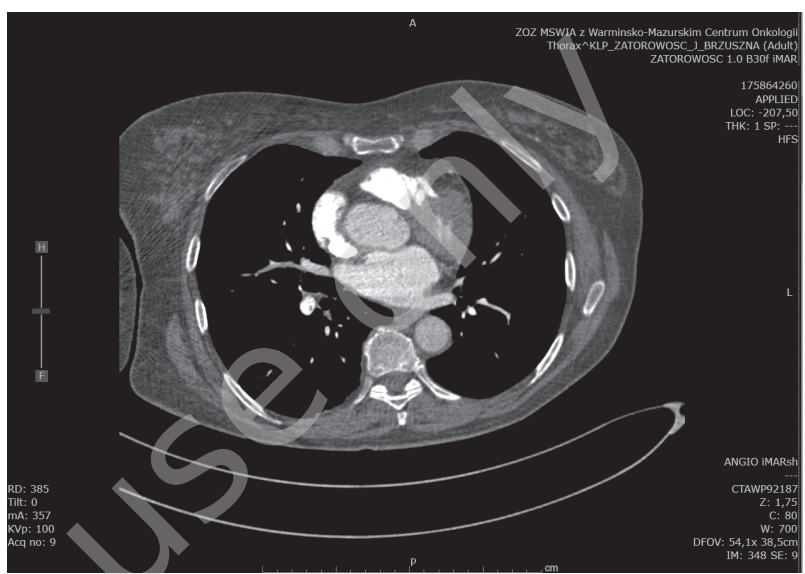

\section{DISCUSSION}

As mentioned at the beginning, hormonal therapy is still a mainstay of treatment in patients with estrogen-positive-receptor $(E R+)$ advanced breast cancer. Managing the negative effects of estrogen on breast cancer cells can be achieved by using several classes of drugs, of which tamoxifen and aromatase inhibitors (Al) are the most commonly used. The groundbreaking drug of modern oncology, tamoxifen, is one of the selective estrogen receptor modulators (SERMs), which blocks the ability of estrogens to stimulate ER while Al act by inhibiting the peripheral conversion of androgens to estrogens.

In patients with advanced breast cancer after menopause and with high ER and PgR receptors expression, induction hormone therapy with $\mathrm{Al}$ (e.g. letrozole) can be used. Tamoxifen, in turn, may be considered for pre-menopausal patients and when contraindications to Al exist (e.g. advanced osteoporosis).

In the presented case ductal breast cancer with ER and PgR receptors expression was diagnosed and the letrozole, a drug from the group of Al, was included. In this type of cancer Al effectiveness is higher when compared to tamoxifen in both the risk of relapse and the incident of death. The side effects of Al are associated with accelerating osteopenia and osteoporosis which can lead to pathological bone fracture [6, 7]. Osteporotic fracture of the humerus and disease progression that occurred in the case presented above were a strong recommendation to switch letrozole therapy to tamoxifen.

Despite the well-demonstrated anti-neoplasmatic efficacy of tamoxifen, some studies revealed its serious side effects. It is known that tamoxifen increases the risk of not only endometrial 
cancers but also the risk of thromboembolic complications, including life threatening incidents such as PE, DVT and cerebrovascular events $[6,8]$.

Monotherapy with tamoxifen is associated with a $2-3 \%$ risk of VTE, and this risk increases with patient's age; breast cancer postmenopausal women are three-fold more likely to develop VTE than patients before menopause. It should be noted that Al are associated with VTE less frequently than tamoxifen; however, the risk of VTE is still significantly higher than in the healthy female population who did not undergo such therapy $[9,10]$.

The mechanism of the above mentioned side effects of tamoxifen is not entirely understood. As tamoxifen can act as a partial estrogen-agonist, it resembles the effect of estrogen in postmenopausal woman. One of the pathways of its procoagulant effect is reduction of antithrombin and protein S concentrations [11]. In few trials with postmenopausal patients with estrogen intake, an increase in prothrobmin fragment $1+2$ was observed $[12,13]$. Both the prothrombin fragment $1+2$ increase and reduction in the level of protein $\mathrm{S}$ by tamoxifen might reflect the reduced anticoagulant activity, and also enhance the risk of VTE $[14,15]$. Some experimental studies claim that tamoxifen increases the intimal thickness in arteries and, when taken continuously, it promotes endothelial proliferation, which predisposes to thrombus formation $[16,17]$. Tamoxifen also activates phospholipase $C \gamma$, $\mathrm{PI} 3$-kinase and NADPH oxidase pathway that causes the release of $\mathrm{Ca}^{2+}$ from the endoplasmic reticulum causing the influx of $\mathrm{Ca}^{2+}$ into the platelets and hence activating them [16].

Among cancer patients PE (which can be a consequence of VTE) is often diagnosed incidentally during imaging aimed at diagnosing and monitoring the progression of neoplastic disease. It is estimated that PE is the second leading cause of death in patients with active malignancy and when left untreated, can have a mortality rate of up to $30 \%$ [18]. Potentially lethal courses of PE require special diagnostic care, particular in patients with additional VTE risk factors.

In the case presented above, we diagnosed intermediate-risk $\mathrm{PE}$ and according to ESC current guidelines for the diagnosis and management of the acute PE therapy with low-molecular-weight-heparin (enoxaparin) was started.

The necessary clinical problem to solve is the issue of prophylactic antithrombotic treatment, which should be used in patients with advanced breast cancer who have an increased risk of VTE due to implemented hormonal therapy.
In everyday clinical practice scale developed by Khorana et al. [19] is commonly used to assess the thromboembolic risk in cancer outpatients starting chemotherapy. This scale is based on the tumor type, hemoglobin concentration or use of erythropoietin stimulating agents, white blood cell count, platelet count, and Body Mass Index (BMI). Unfortunately, the algorithm does not include some malignancies (e.g. breast cancer) and same patients may have an underestimated risk of VTE using the above scale. The patient described by us belonged to this "problematic" group: in the Khorana risk scale (KRS), had 0 points, i.e. a low risk of VTE.

Current international guidelines do not recommend routine pharmacological thromboprophylaxis in ambulatory cancer patients [20,21]. In these recommendations, use of VTE prevention is recommended only for patients requiring hospital treatment, undergoing surgery, those with pancreatic cancer, treated with immunomodulatory drugs combined with steroids or other systemic anticancer therapies, and who are ambulatory receiving systemic anticancer therapy at intermediate-to-high risk of VTE assessed by KRS [22].

In 2017 Gerotziafas et al. [23] described the new COMPASS-CAT (tab. 2) score dedicated to outpatients risk of VTE during cancer chemotherapy. It should be emphasised that the largest group of patients in the COMPASS-CAT derivation cohort were breast cancer patients (61\%). The risk factors included in the algorithm were among others: the use of anthracycline or hormone therapy, the presence of catheters in the central veins, cancer advancement and the presence of cardiovascular risk factors. The sensitivity and specificity of this risk assessment model for VTE prediction were $88 \%$ and $52 \%$, respectively.

TABLE 2.

Simplified COMPASS CAT SCORE for VTE prediction among ambulatory cancer patients. According to Gerotziafas et al. [23].

\begin{tabular}{|c|c|}
\hline \multicolumn{2}{|c|}{ COMPASS-CAT Score } \\
\hline PREDICTION FACTORS FOR VTE & SCORE \\
\hline Cancer related factors & 6 \\
\hline Anti-hormonal therapy/anthracycline treatment & 4 \\
\hline Time since diagnosis $\leq 6$ months & 3 \\
\hline Central venous catheter & 2 \\
\hline Advanced stage of cancer & 5 \\
\hline $\begin{array}{c}\text { Predisposing risk factors } \\
\text { history of peripheral artery disease, ischemic stroke, } \\
\text { hypertension, hyperlipidemia, diabetes, obesity, } \\
\text { coronary artery disease) }\end{array}$ & 1 \\
\hline $\begin{array}{c}\text { Recent hospitalization for acute medical illness } \\
\text { Personal history of VTE }\end{array}$ & 5 \\
\hline
\end{tabular}




\begin{tabular}{|c|c|}
\hline \multicolumn{2}{|c|}{ COMPASS-CAT Score } \\
\hline Biomarkers & \\
\hline Platelets count $\geq 350 \times 109 / \mathrm{L}$ & 2 \\
\hline Score $0-6$ low/intermediate risk; Score $\geq 7$ high risk \\
\hline
\end{tabular}

COMPASS-CAT - Prospective Comparison of Methods for thromboembolic risk assessment with clinical Perceptions and AwareneSS in real life patients-Cancer Associated Thrombosis, VTE venous thromboembolism.

Using the COMPASS-CAT scale, the patient presented by us would receive 8 points and therefore belonged to the group of high risk of VTE in which thromboprophylaxis with LMWH is indicated.

Considering the patient's history of reduced activity in recent months due to bone pain caused by osteoporosis after letrozole and the use of radiation therapy as well as surgical treatment of the fracture of the humerus carried out without VTE prophylaxis (which was in line with current VTE prophylaxis recommendations) could be added on the COMPASS scale - CAT another 5 points - this would give a total of 13 out of 28 points. Thus, the patient we presented was a high-risk patient who should be considered for the prophylaxis of VTE, perhaps already at the stage of activity reduction, and certainly after the fracture of humerus. In this case, the patient's risk should be reevaluated based on the COMPASS-CAT scale, especially that the aromatase inhibitor was switched to tamoxifen, whose thrombotic potential is significantly higher compared to the letrozole used so far (3.8\% vs. $2.0 \%$, respectively, $p<0.001$ ) [24].

It is worth pointing out that according to the latest recommendations of The International Initiative on Thrombosis and Cancer (ITAC) the COMPASS-CAT model scale is especially dedicated to the patients with breast cancer [22]. It should be noted that only the small number of outpatients with frequent cancers such as breast cancer were included in clinical trials for primary prevention of VTE, as did the creation of the most common VTE risk scales (only about $6.6 \%$ of patients are patients with breast cancer). Predictive scores: KRS, PROTECHT, CONKO score, Vienna CATS Score, are recommended for the cancer patients starting chemotherapy [25].
It seems that the indications for the use of primary VTE prevention in cancer patients should be periodically reevaluated based on the risk-benefit model each time the cancer therapy is changed, when hormone therapy with a higher risk of thrombosis is administered, or with a decrease in patient mobility. Randomized studies on which the recommendations are created, based the VTE risk assessment on the KRS (patients with moderate to high risk VTE), while the basis for this scale was retrospective assessment, and patients with haematological cancers were a large group in the assessed cohort. Meanwhile, the risk of cancer-associated thrombosis (CAT) changes during chemo- and hormonal therapy, and initially low-risk outpatients, after using scales dedicated to underestimated in KRS types of cancer, may be qualified for VTE prophylaxis as high-risk patients, despite not meeting the typical conditions contained in the recommendations (just like it did in the case we describe).

An open-ended question is if we should use only KRS for the risk stratification or the VTE risk should be reevaluated using scales dedicated to specific type of cancer (e.g. breast cancer) like COMPASS-CAT scale and when this stratification should be performed (only at the beginning or during chemotherapy).

The above question is clinically relevant, while CAT has many consequences including increased morbidity, bleeding complications, increased risk of recurrent VTE and cancer treatment delays [26].

\section{CONCLUSIONS}

Deep vein thrombosis and pulmonary embolism are a serious issues among breast cancer patients, especially in those treated with tamoxifen. That's why every single case should be investigated closely for the primary thromboprophylaxis. VTE prediction risk scales dedicated to specific types of cancer may be particularly useful in such cases (e.g. COMPASS-CAT Score for breast cancer patients)

\section{References}

1. Tagalakis V, Patenaude V, Kahn SR et al. Incidence of and mortality from venous thromboembolism in a real-world population: the Q-VTE Study Cohort. Am J Med. 2013; 126(9): 832.e13-21.

2. Chew HK, Wun T, Harvey D et al. Incidence of Venous Thromboembolism and Its Effect on Survival Among Patients With Common Cancers. Arch Intern Med. 2006; 166(4): 458-64.

3. Brand JS, Hedayati E, Bhoo-Pathy N et al. Time-dependent risk and predictors of venous thromboembolism in breast cancer patients: A population-based cohort study. Cancer. 2017; 123(3): 468-75.

4. Prandoni P, Falanga A, Piccioli A. Cancer and venous thromboembolism. Lancet Oncol. 2005; 6: 401-10.

5. Braithwaite RS, Chlebowski RT, Lau J et al. Meta-analysis of vascular and neoplastic events associated with tamoxifen. J Gen Intern Med. 2003; 18(11): 937-47. 
6. Metzger Filho O, Giobbie-Hurder A, Mallon E et al. Relative Effectiveness of Letrozole Compared With Tamoxifen for Patients With Lobular Carcinoma in the BIG 1-98 Trial. J Clin Oncol. 2015; 33(25): 2772-9.

7. Burstein HJ, Temin S, Anderson H et al. Adjuvant endocrine therapy for women with hormone receptor-positive breast cancer: American society of clinical oncology clinical practice guideline focused update. J Clin Oncol. 2014; 32(21): 2255-69.

8. Wojtukiewicz MZ, Sierko E. Zakrzepy a nowotwory. In: Windyga J,Pasierski T, Torbicki A (ed). Zakrzepy i zatory. Wyd. I. Wydawnictwo Lekarskie PZWL, Warszawa 2014: 85-105.

9. Wojtukiewicz MZ, Sierko E. Powikłania zakrzepowo zatorowe u chorych na nowotwory. In: Wojtukiewicz MZ, Deptała A (ed). Problemy hematologiczne u chorych na nowotwory. Wyd. I. Termedia, Poznań 2014; 225-50.

10. Cushman M, Costantino JP, Bovill EG et al. Effect of tamoxifen on venous thrombosis risk factors in women without cancer: the Breast Cancer Prevention Trial. Br J Haematol. 2003; 120: 109-16.

11. Caine YG, Bauer KA, Barzegar S et al. Coagulation activation following estrogen administration to postmenopausal women. Thromb Haemost. 1992; 68: 392-5.

12. de Valk-de Roo GW, Stehouwer CD, Meijer P et al. Both raloxifene and estrogen reduce major cardiovascular risk factors in healthy postmenopausal women: a 2-year, placebo-controlled study. Arterioscler Thromb Vasc Biol. 1999; 19: 2993-3000.

13. Kessler CM, Szymanski LM, Shamsipour Z et al. Estrogen replacement therapy and coagulation: relationship to lipid and lipoprotein changes. Obstet Gynecol. 1997; 89: 326-31.

14. Zoller B, Garcia de Frutos P, Dahlback B. Evaluation of the relationship between protein S and C4b-binding protein isoforms in hereditary protein S deficiency demonstrating type I and type III deficiencies to be phenotypic variants of the same genetic disease. Blood. 1995; 85: 3524-31.

15. De Pinho Pessoa BB, Menezes Cavalcante BB, Maia MP et al. Effect of tamoxifen on arterial microvascular anastomosis. Microsurgery. 2007; 27: 286-8.

16. Ceran C, Aksam E, Aksam B et al. Tamoxifen-related thrombosis: an experimental study in rat venous microvascular anastomosis model. Ann Plast Surg. 2017; 78: 213-6.

17. Bĕlohlávek J, Dytrych V, Linhart A et al. Pulmonary embolism, part I: Epidemiology, risk factors and risk stratification, pathophysiology, clinical presentation, diagnosis and nonthrombotic pulmonary embolism. Exp Clin Cardiol. 2013; 18: 129-38.

18. Khorana AA, Kuderer NM, Culakova E et al. Development and validation of a predictive model for chemotherapy-associated thrombosis. Blood. 2008; 111(10): 4902-7.

19. Lyman GH, Bohlke K, Khorana AA et al. Venous thromboembolism prophylaxis and treatment in patients with cancer: American Society of Clinical Oncology clinical practice guideline update 2014. J Clin Oncol. 2015; 33: 654-6.

20. NCCN Clinical Practice Guidelines in Oncology. Version 2, 2018.

21. Farge D, Frere C, Connors J et al. 2019 international clinical practice guidelines for the treatment and prophylaxis of venous thromboembolism in patients with cancer. Lancet Oncol. 2019; 10(20): e566-81.

22. Gerotziafas GT, Taher A, Abdel-Razeq H et al. A predictive score for thrombosis associated with breast, colorectal, lung or ovarian cancer, the prospective COMPASS-cancer associated thrombosis study. Oncologist. 2017; 22: 1222-31.

23. Coates AS, Keshaviah A, Thurlimann B et al. Five years of letrozole compared with tamoxifen as adjuvant therapy for postmenopausal women with endocrine-responsive early breast cancer. Update of study BIG 1-98 J Vlin Oncol. 2007; 25: 486-92.

24. van Es N, Di Nisio M, Cesarman G et al. Comparison of risk prediction scores for venous thromboembolism in cancer patients: a prospective cohort study. Haematologica. 2017; 102(9): 1494-501.

25. Lyman GH. Venous thromboembolism in the patient with cancer: focus on burden of disease and benefits of thromboprophylaxis. Cancer. 2011; 117(7): 1334-49.

Authors' contributions: Agata Tomaszewska: 40\%; Małgorzata Żach-Muzolf: 30\%; Radosław Grabysa: 30\%.

Financial support:

None.

Ethics:

The authors had full access to the data and take full responsibility for its integrity.

All authors have read and agreed with the content of the manuscript as written.

The paper complies with the Helsinki Declaration, EU Directives and harmonized requirements for biomedical journals. 